\title{
LOCAL MEASURES DISTRIBUTION FOR THE ESTIMATION OF THE ELONGATION RATIO OF THE TYPICAL GRAIN IN HOMOGENEOUS BOOLEAN MODELS.
}

\author{
Tatyana Eremina ${ }^{\bowtie}$, Johan Debayle, Frédéric Gruy and Jean-Charles Pinoli \\ MINES Saint-Etienne, CNRS, UMR 5307 LGF, Centre SPIN, 158 cours Fauriel, SAINT-ETIENNE Cedex 2 \\ 42023, France \\ e-mail: tatyana.eremina@emse.fr,debayle@emse.fr,fgruy@emse.fr,pinoli@emse.fr \\ (Received April 2, 2021; revised June 1, 2021; accepted June 1, 2021)
}

\begin{abstract}
We introduce a particular localization of the Minkowski functionals to characterize and discriminate different random spatial structures. The aim of this paper is to present a method estimating the typical grain elongation ratio in a homogeneous Boolean model. The use of this method is demonstrated on a range of Boolean models of rectangles featuring fixed and random elongation ratio. An optimization algorithm is performed to determine the elongation ratio which maximize the likelihood function of the probability density associated with the local perimeter measure. Therefore, the elongation ratio of the typical grain can be deduced.
\end{abstract}

Keywords: Boolean Model, elongation ratio, local measures, Minkowski functionals.

\section{INTRODUCTION}

The structure of granular media - as could be encountered, for example, in materials and chemical sciences - is a complex concept from the geometric and topological point of view. Some spatial configurations occurring in natural or industrial processes could be modeled by random closed sets (RACS), as was first suggested by Matheron (1975). Thereby Stochastic Geometry provides quantitative descriptors to control and quantify the processes. The purely probabilistic characteristics (as Choquet capacity and correlation functions (Ballani, 2007; Molchanov and Stoyan, 1994)) are generally difficult to connect to the geometric characteristics of the random set. Complete characterization of the effective morphology requires knowledge of an infinite set of $n$-point statistical correlation functions (Arns et al., 2002). On the other hand, Integral Geometry (Mecke, 2000; Santaló, 1976) offers a complete system of morphological descriptors - the Minkowski functionals (MFs) (with different normalization they are also referred to as intrinsic volumes or quermassintegrals). Indeed, the Hadwiger's characterization theorem (Hadwiger, 1957; Klain and Rota, 1997) states that all morphological information is essentially contained in this family of MFs, see e.g. Sevink (2007). In $\mathbb{R}^{2}$ they coincide up to normalization with classical geometric measurements: Euler-Poincaré characteristic $V_{0}(\cdot)$, perimeter $V_{1}(\cdot)$ and area $V_{2}(\cdot)$. Thus the study of the probability distributions of MFs of the random closed sets gives access to the morphology description on different levels (global - of the entire structure, local - in the neighbourhood of a point, or individual - morphology of the typical grain).

The main contribution of this paper is to show how the local measures could be used to distinguish the typical grain morphology in a planar germ-grain model - otherwise inaccessible due to the superimposition of grains. A method based on the maximum likelihood estimation of the probability density functions of local measures has been developed to estimate the "elongation ratio" of the typical grain in a germ-grain model. In the first section the geometric measures on the random closed sets and their localizations are introduced. In the next section we describe a particular system of the planar Boolean models of rectangles. Two types of random rectangles will be of interest - with fixed and random elongation ratio. From there on the realizations of the Boolean model we demonstrate the results on the elongation ratio estimation. The final section is devoted to simulation results of the elongation ratio estimation for Boolean model of rectangles.

\section{CHARACTERIZATION OF RACS VIA MINKOWSKI FUNCTIONALS}

The Minkowski functionals (MF) play an important role in the geometrical characterization of spatial structures, quantifying certain properties such as volume, surface area or mean width. These functionals are also known as the intrinsic volumes 
$V_{0}, \ldots, V_{d}$. They could be defined as coefficients of Steiner polynomials. For a set $A \subset \mathbb{R}^{d}$ and $r>0$, the volume of its $r$-parallel set $A_{\oplus r}=\left\{x \in \mathbb{R}^{d}\right.$ : $\left.\inf _{y \in A}\|x-y\| \leq r\right\}$ is a polynomial in $r$ :

$$
\mathscr{H}^{d}\left(A_{\oplus r}\right)=\sum_{s=0}^{d} b_{d-s} V_{s}(A) \cdot r^{d-s}, \quad \text { (Steiner formula) }
$$

where $\mathscr{H}^{d}$ is the $d$-dimensional Hausdorff measure and $b_{d-s}$ is the volume of $(d-s)$-dimensional unit ball in $\mathbb{R}^{d}$.

The global characteristics of a random closed set $\tilde{\Xi}$ could be assessed by asymptotic values of Minkowski functionals - the specific intrinsic volumes:

$$
\bar{V}_{s}(\tilde{\Xi})=\lim _{n \rightarrow \infty} \frac{\mathbb{E} V_{s}(\tilde{\Xi} \cap n W)}{V_{d}(n W)}
$$

This limit exists for each $s=0, \ldots, d$ and for any sequence $\{n W\}$ of compact convex observation windows such that $V_{d}(W)>0$ and the origin of $\mathbb{R}^{d}$ is in $W$, see Schneider and Weil (2008, Theorem 9.2.1). The functional $\bar{V}_{s}(\tilde{\Xi})$ is called the $s$-th specific intrinsic volume of $\tilde{\Xi}$. For detailed introduction see Schneider and Weil (2008).

However, the global measurements are not always suitable for the description of a complex spatial structure. For example, in Fig.1 two spatial structures possess the same global area, perimeter and EulerPoincaré characteristic, while presenting strongly different morphologies.

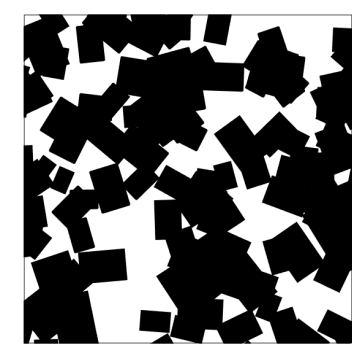

(a)

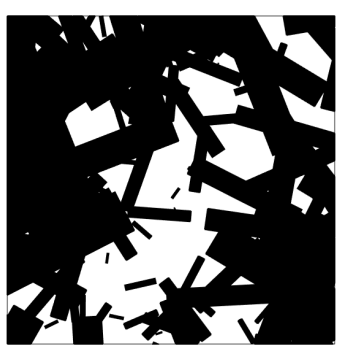

(b)
Fig. 1: Realizations of two homogeneous Boolean models of rectangles. The global measures remain equal (equal MF densities - area density, perimeter density and specific Euler-Poincaré characteristic), and equal intensity of the underlying point process.

To overcome this stiffness and get finer geometric characterization of a complex random spatial structure different extensions of MFs were developed in the literature. Among them are the curvature measures (Schneider, 2014), MFs of parallel sets to discriminate morphologies (Arns et al., 2002), first and second order properties of MFs (Rahmani et al., 2017), combinations of MFs - shape diagrams (Presles et al., 2012).

\section{LOCALIZATION OF MINKOWSKI FUNCTIONALS}

This paper is concentrated on a particular localization of MFs - random measures induced by a structure $\tilde{\Xi}$ for any Borel set $A \in \mathscr{B}_{\mathbb{R}^{d}}$ (i.e. the Borel $\sigma$-algebra):

$$
\begin{aligned}
& \mathscr{H}^{s}(\tilde{\Xi} \cap A), s=0, \ldots, d, \\
& \mathscr{H}^{s}-s \text {-dimensional Hausdorff measure in } \mathbb{R}^{d} .
\end{aligned}
$$

For the discussion on the measurability of the random variables $\mathscr{H}^{s}(\tilde{\Xi})$ see Zähle (1982); Baddeley and Molchanov (1997)..

Thus, the Minkowski measures (2) are the restrictions of the Minkowski functionals to Borel sets, allowing a local analysis of the structure (Legland et al., 2007; Pinoli, 2014, p. 110). Here the set $A$, referred to as the test set. The local measures of the random closed set, as considered in this paper, admit two degrees of stochasticity: one for the random set to be characterized and the other for the location of the test set. Formally speaking, let $\tilde{\Xi}$ be a $d$-dimensional random closed set $\tilde{\Xi}: \Omega \rightarrow \mathbb{F}$ and $\tilde{x}$ be a random point $\tilde{x}: \Omega^{\prime} \rightarrow \mathbb{R}^{d}$. Then local measures are determined for a random pair:

$$
(\tilde{\Xi}, \tilde{x}):\left(\Omega \times \Omega^{\prime}\right) \rightarrow\left(\mathbb{F} \times \mathbb{R}^{d}\right) .
$$

Further, we would refer to this pair as main set $\tilde{\Xi}$ and $\tilde{x}$ will determinate the location of a test set. The number of realizations of a random set $\tilde{\Xi}$ is denoted by $N$ and the number of locations of a test set by $n_{l o c}$.

The descriptors for the geometric characterization of a spatial structure (i.e. the random closed set $\tilde{\Xi}$ ) are then the probability distributions of the values taken by the Minkowski measures (2) on the realizations of a random pair $(\tilde{\Xi}, \tilde{x})$.

Few theoretical advancements on the local measures of the random closed sets could be found in the literature. The asymptotic values of the local measures are given by the specific intrinsic volumes (1). If we restrict $\tilde{\Xi}$ to be a Boolean model (Chiu et al., 2013, ch. 3) (union set of a uniformly distributed realizations of a random closed set - typical grain), then the MF densities can be used to obtain the estimators of the expectation of MFs of the typical grain and also of the Boolean model intensity using so called Davy-Miles' formulae (Miles, 1976). In other 
words, under suitable assumptions, the observable quantitative descriptors (Minkowski functionals) of the union set allow us to obtain estimators for the certain parameters of the underlying particle process (Weil, 2001; Schneider and Weil, 2008).

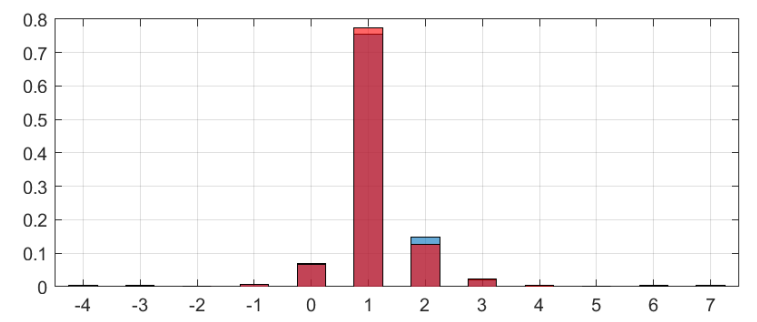

(a) local Euler-Poincaré measure

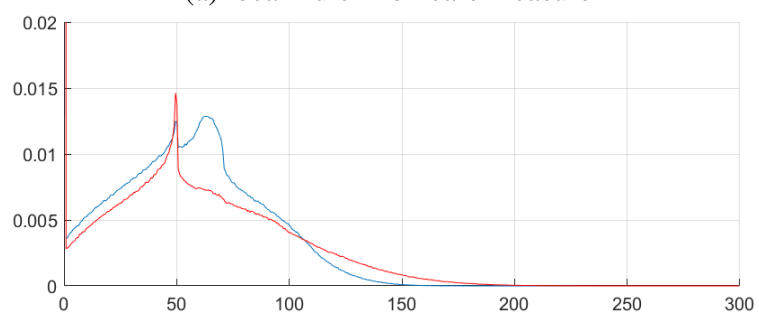

(b) local perimeter measure

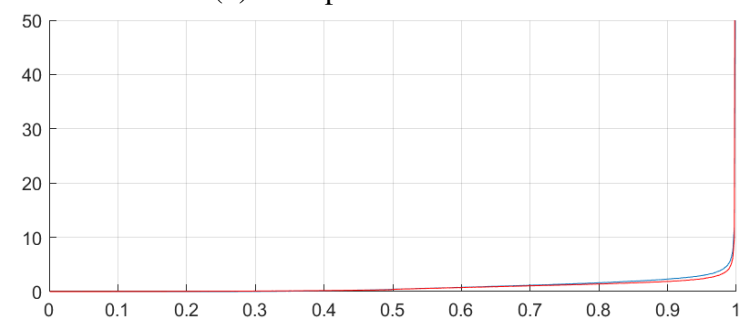

(c) local area measure normalized by the area of a test set

Fig. 2: Probability mass function and probability density functions of the local measures compared for BMs of rectangles in Fig. (1a) in blue and (1b) in red.

Localizations of that type show a particular interest in materials science. The first generalized Minkowski measure - Euler-Poincaré characteristic, showed to be highly correlated to the percolation (for a study on percolation in Boolean models see Scholz et al. (2015), study of a local porosity distribution (Hilfer, 2000), percolation in a Fontainebleau sandstone (Arns et al., 2005)).

Let the test set be an open ball with a radius $r>0$ centered in a random point $\tilde{x} \in \mathbb{R}^{d}$. We concentrate on the random measures induced by localizations of Minkowski functionals. The $(d-1)$-th and $d$-th Minkowski measures (2) are written as:

$$
\begin{aligned}
V_{d-1}(\tilde{\Xi}, \tilde{x}) & =\mathscr{H}^{d-1}\left(\partial \tilde{\Xi} \cap B_{r}(\tilde{x})\right) ; \\
V_{d}(\tilde{\Xi}, \tilde{x}) & =\mathscr{H}^{d}\left(\tilde{\Xi} \cap B_{r}(\tilde{x})\right) .
\end{aligned}
$$

They will be further referred to as the local perimeter measure (4) and the local area measure (5) respectively. Note, that for one realization $x$ of $\tilde{x}$ these local measures are straightforward generalizations of Minkowski functionals. Indeed, they are the restriction of the MFs to a particular set $B_{r}(x)$. Consider a very large random structure $\tilde{\Xi}$, and for its every realization $\Xi$ take values of the Minkowski functionals for $\Xi \cap$ $B_{r}(\tilde{x})$. For different positions of test set (i.e. different $\tilde{x})$ different values are obtained, which provide $d$ measures in the sense of the measure theory: mappings which assign real numbers to sets and which become "random measures" for random structures, see Chiu et al. (2013, ch. 7) for an introduction.

An example of the constructed descriptors $V_{s}(\tilde{\Xi}, \tilde{x})$, $s=0,1,2$ in $\mathbb{R}^{2}$ is showed on Fig. 2 . The probability mass function $(s=0)$ and the probability density functions $(s=1,2)$ of local measures are depicted here for two particular random sets. The local Euler-Poincaré measure 2a has a discrete probability distribution and takes entire values due to the nature of the Euler-Poincare characteristic. The local perimeter measure $2 \mathrm{~b}$ is a continuous non-negative real-valued random variable. Due to the choice of a particular test set this pdf shows a peak at 0 (for some realizations of $\tilde{x}$ the test set entirely lies in the main set $\tilde{\Xi}$ ) and intermediate peaks which correlate with the size of the test set. The local area measure $2 \mathrm{c}$ is a continuous positive real-valued random variable, once normalized by the area of a test set, it takes value in $] 0,1], 1$ when test set falls entirely into a main set $\tilde{\Xi}$.

The expectations of the local measures $V_{S}(\tilde{\Xi}, \tilde{x})$ in the case of statistical homogeneity are given (Arns et $a l ., 2005$ ) by the product of the $d$-dimensional volume $V_{d}\left(B_{r}(\tilde{x})\right)=b_{d} r^{d}$ of the test set $B_{r}(\tilde{x})$ and a constant dependent on $\tilde{\Xi}$.

$$
\mathbb{E} V_{s}(\tilde{\Xi}, \tilde{x})=b_{d} r^{d} \bar{V}_{s}(\tilde{\Xi}), \quad s=0, \ldots, d
$$

In $\mathbb{R}^{2}$ - perimeter density $\bar{V}_{1}(\tilde{\Xi})$ and area density $\bar{V}_{2}(\tilde{\Xi})$ for the local perimeter measure (4) and the local area measure (5) respectively.

Thus the expectation of the local measures, yet giving the first information about $\tilde{\Xi}$, does not determine its distribution. For a more complex spatial structure as a Boolean model, the typical grain morphology could not be accessed using the global MFs, neither by first moments of the local measures, as they depend only on the global MFs. That is why the distributions of local measures (4)-(5) for the Boolean model are studied. This methodology has been previously studied by Arns et al. (2005) to characterize the Fontainebleau sandstone. 


\section{LOCAL MEASURES OF BOOLEAN MODEL OF RECTANGLES}

A Boolean model (BM) is particular random closed set defined as (see e.g. Baddeley et al. (2006) and references therein):

$$
\tilde{\Xi}=\bigcup_{i=1}^{\infty}\left(z_{i}+Z_{i}\right)
$$

where the points (germs) $z_{i}$ belong to a stationary Poisson process in $\mathbb{R}^{d}$ of intensity $\lambda$, while the grains $Z_{i}$ are i.i.d. random compact sets in a mark space $\mathbb{K}$, independent from the germs $z_{i}$. The typical grain of the Boolean model is a random closed set $Z_{0}$ with the same distribution as $Z_{i}$. The typical grain could be suitably parameterized $Z_{0}=Z_{0}(\tilde{s})$. For instance, in a case of a Boolean model with spherical grains $\mathbb{K}=\mathbb{R}_{+}$and $\tilde{s}$ is the radius of a random ball; in a case of rectangular grains $\mathbb{K}=\mathbb{R}_{+} \times \mathbb{R}_{+} \times[0,2 \pi[$ and $\tilde{s}=(A, B, \phi)$ where $A$ and $B$ are the random length and width, $\phi$ is the random orientation of the rectangle which left-bottom corner is at the origin; etc. Note that the Boolean model can be introduced in a more general way with grains only supposed to be compact, the characterization of such a model will not be our objective here.

We compare the sensitivity of the local measures to deviations in the elongation ratio of the typical grain. To do this the homogeneous Boolean models $\tilde{\Xi}$ of rectangles with different elongations are considered. They are constructed in such a way that the global measures $\bar{V}_{0}(\tilde{\Xi}), \bar{V}_{1}(\tilde{\Xi}), \bar{V}_{2}(\tilde{\Xi})$ keep the same values.

In the first dataset the typical grain is a randomly oriented rectangle of gamma-distributed width and fixed elongation ratio $e=1.5, \ldots, 10$. One realization of a homogeneous Boolean model of rectangles with fixed elongation ratio gives one simulated spatial structure. The exact parameters are listed further, see Eq. (11)-(14). The examples from this dataset could be seen in Fig. 3 (1st row).

In the second dataset, the typical grain is a randomly oriented rectangle of gamma-distributed width and random elongation ratio - log-normally distributed with $\mathbb{E} \tilde{e}=1.5, \ldots, 10$ and $\mathbb{V} \tilde{e}=0.25$. For each rectangle an elongation ratio is generated from the log-normal distribution with the corresponding expectation. The elongation ratio distributions are depicted in Fig. 5. A homogeneous Boolean model of rectangles with random elongation ratio is obtained. Its parameters are listed in Eq. (11)-(16). Some examples of the BM realizations are represented in Fig. 4 (1st row).

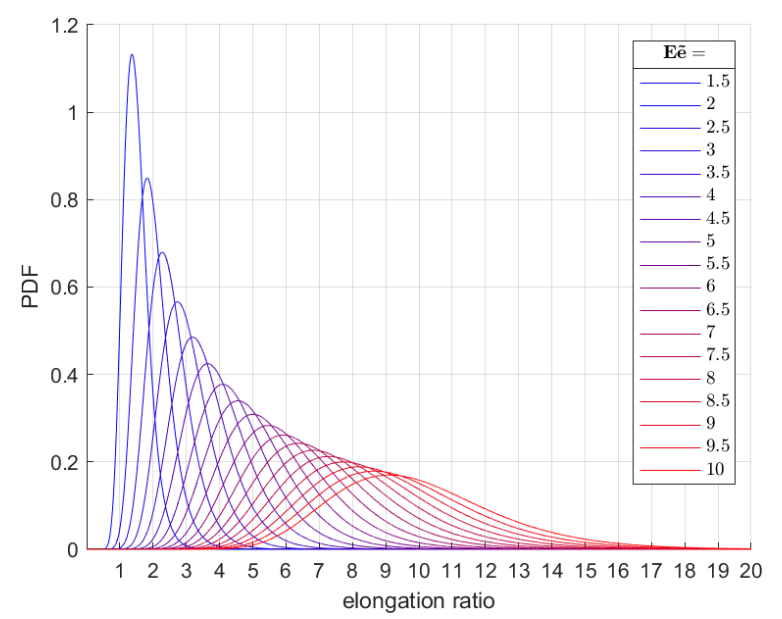

Fig. 5: Log-normal distributions for the elongation ratio of rectangular grains in the Boolean models. $\mathbb{E} \tilde{e}=1.5,2, \ldots, 9.5,10$ (blue $\rightarrow$ red $)$.

The BM realizations in Fig. 1 are taken from the first dataset with fixed elongation ratio. As shown in Fig. 2a and Fig. 2c the local Euler-Poincaré and local area measures are quite similar for this range of Boolean models. It is also impossible to distinguish these models using the global MFs - the system (11)-(16) was constructed in a manner that global measures are equal. For example for models in Fig. 1 the reference values are $\bar{V}_{2}(\tilde{\Xi})=0.7, \bar{V}_{1}(\tilde{\Xi})=$ $0.0144, \bar{V}_{0}(\tilde{\Xi})=-7.6998 \times 10^{-5}$. However, the local perimeter measure in Fig. $2 \mathrm{~b}$ indeed allows to distinguish these systems and allows the shape of the typical grain to be estimated. Further, a method based on the local perimeter distribution for estimating the elongation ratio is suggested.

\section{ESTIMATION OF THE ELONGATION RATIO OF THE TYPICAL GRAIN}

We propose a method for estimating the typical grain elongation ratio based on the local perimeter measure distribution. For a one-grain model (simple random closed set - a random disk or a random rectangle) the analytical expression for the local perimeter measure distribution could be obtained directly. However, for complex spatial structures one should have recourse to numerical methods.

To test the sensitivity of local measures to the typical grain elongation ratio $e$ in a homogeneous Boolean model, the global MFs were fixed. Their values are given in Table 1. 


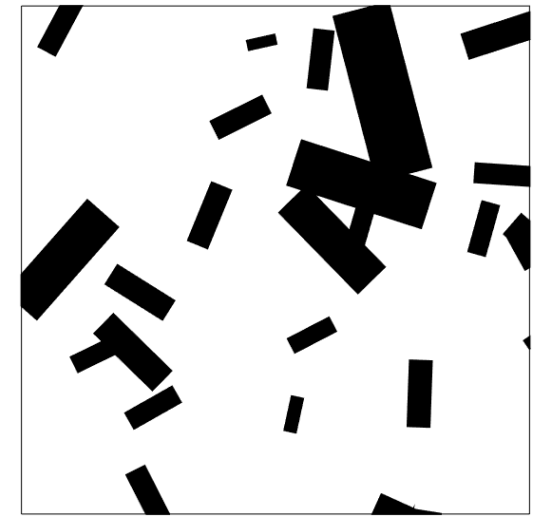

(a) area density 0.2

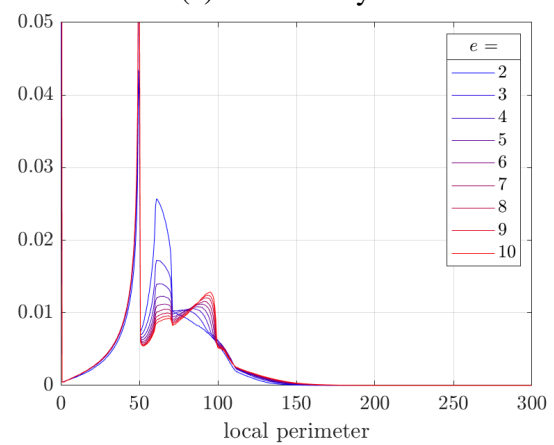

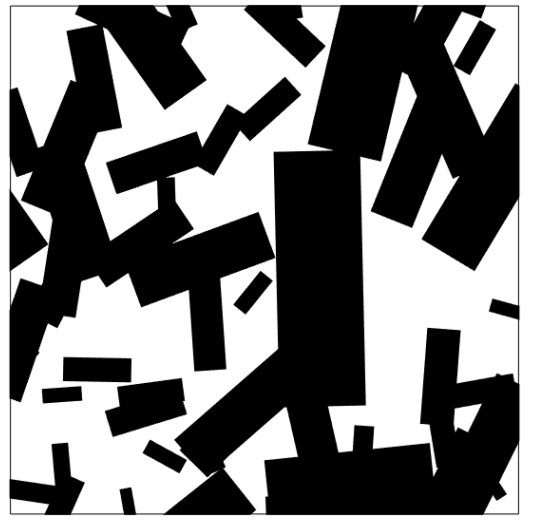

(b) area density 0.4

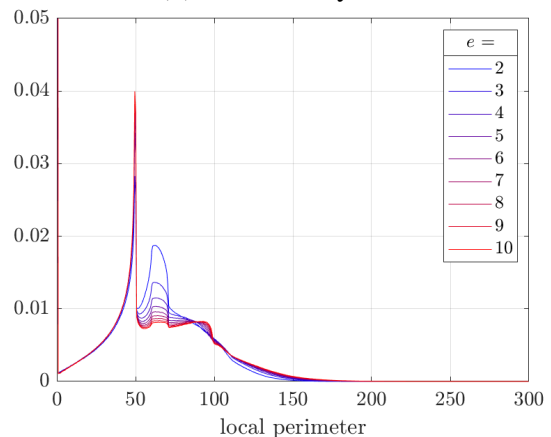

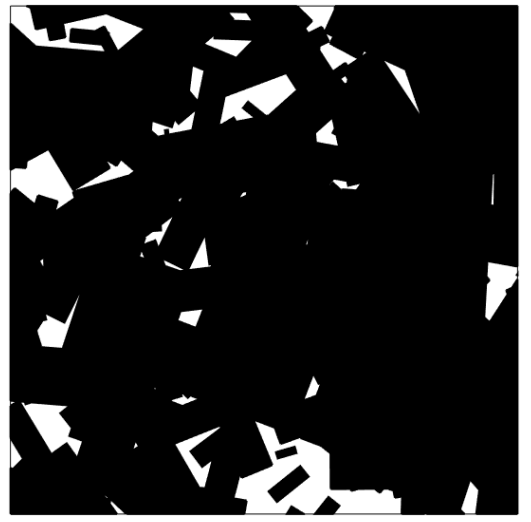

(c) area density 0.9

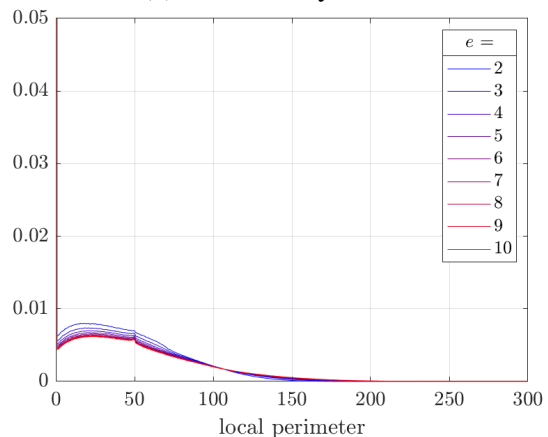

Fig. 3: (1st row) Samples of BMs of rectangles with fixed elongation ratio $e=3$. (2nd row) Probability density functions of the local perimeter measure of BMs of rectangles with the growing typical grain elongation ratio. The distributions are normalized by the total number of samples and the bin width.

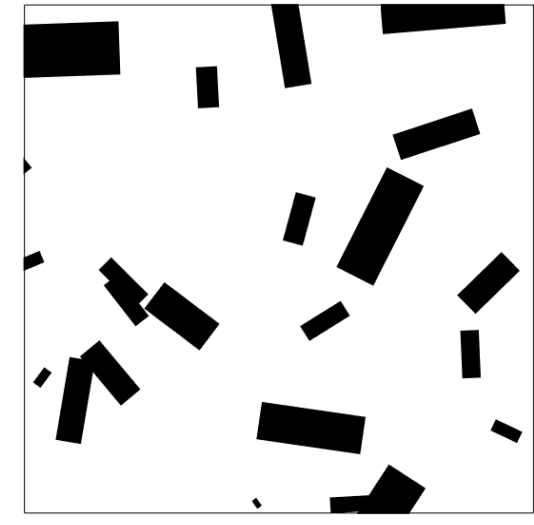

(a) area density 0.2

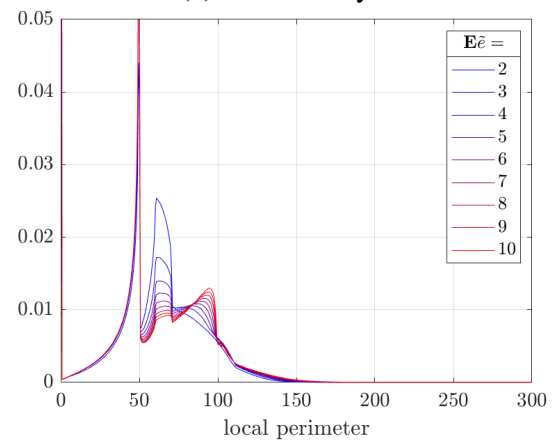

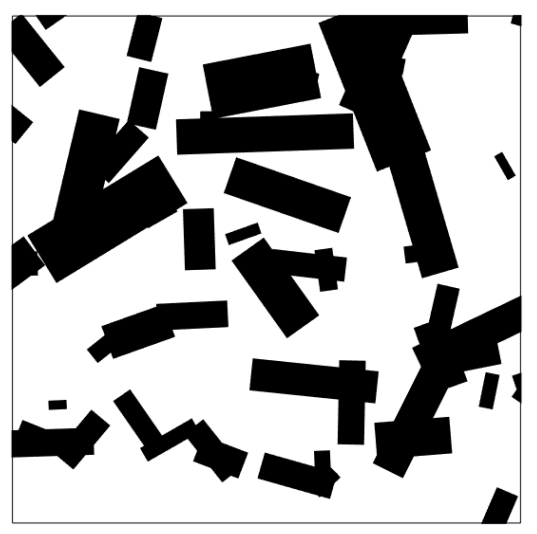

(b) area density 0.4

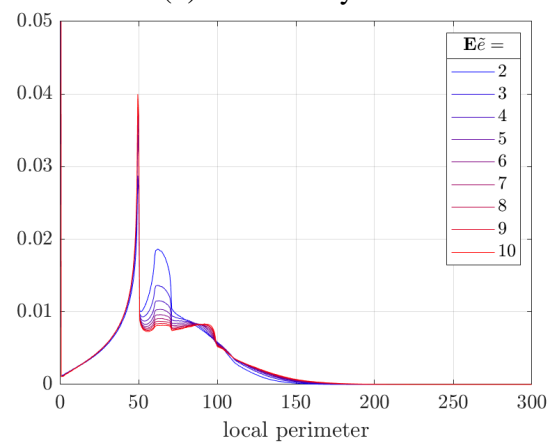

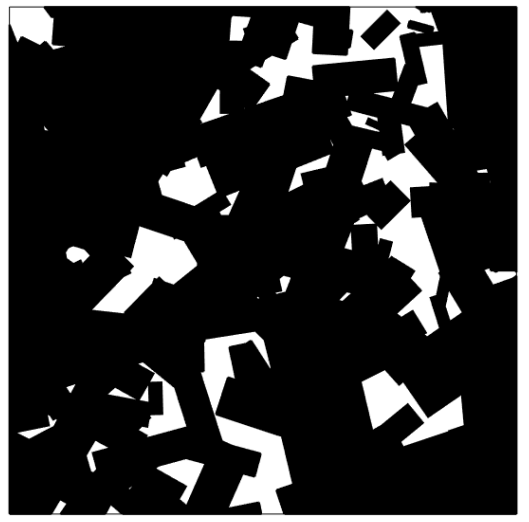

(c) area density 0.9

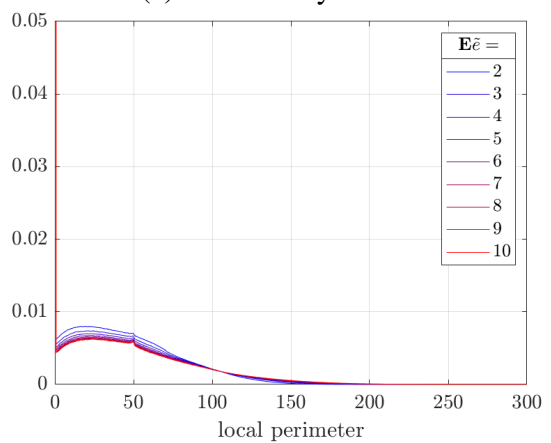

Fig. 4: (1st row) Samples of BMs of rectangles with log-normally random elongation ratio expectation $\mathbb{E} \tilde{e}=3$. (2nd row) Probability density functions of the local perimeter measure of BMs of rectangles with the growing typical grain elongation ratio. The distributions are normalized by the total number of samples and the bin width. 


\begin{tabular}{ccc}
\hline $\bar{V}_{2}(\tilde{\Xi})$ & $\bar{V}_{1}(\tilde{\Xi})$ & $\bar{V}_{0}(\tilde{\Xi})$ \\
\hline 0.2 & 0.0071 & $5.1118 \times 10^{-5}$ \\
0.3 & 0.0100 & $5.4515 \times 10^{-5}$ \\
0.4 & 0.0123 & $4.2860 \times 10^{-5}$ \\
0.5 & 0.0139 & $1.6283 \times 10^{-5}$ \\
0.6 & 0.0147 & $-2.4433 \times 10^{-5}$ \\
0.7 & 0.0144 & $-7.6998 \times 10^{-5}$ \\
0.8 & 0.0129 & $-1.3509 \times 10^{-4}$ \\
0.9 & 0.0092 & $-1.7792 \times 10^{-4}$ \\
\hline
\end{tabular}

Table 1: Fixed values of global MFs for the Boolean models simulation (for both datasets - with fixed and random elongation ratio).

Once the global MFs are fixed, the inverse DavyMiles' formulae gives the intensity value and the MFs of the typical grain:

$$
\begin{aligned}
& \lambda=\left(\frac{\bar{V}_{1}(\tilde{\Xi})^{2}}{\pi\left(1-\bar{V}_{2}(\tilde{\Xi})\right)}+\bar{V}_{0}(\tilde{\Xi})\right) \cdot \frac{1}{1-\bar{V}_{2}(\tilde{\Xi})} ; \\
& \mathbb{E} V_{1}\left(Z_{0}\right)=\frac{\pi \bar{V}_{1}(\tilde{\Xi})\left(1-\bar{V}_{2}(\tilde{\Xi})\right)}{\bar{V}_{1}(\tilde{\Xi})^{2}+\pi \bar{V}_{0}(\tilde{\Xi})\left(1-\bar{V}_{2}(\tilde{\Xi})\right)} ; \\
& \mathbb{E} V_{2}\left(Z_{0}\right)=-\frac{\ln \left(1-\bar{V}_{2}(\tilde{\Xi})\right)}{\lambda} .
\end{aligned}
$$

The intensity $\lambda$ was chosen to fit the densities $\bar{V}_{2}(\tilde{\Xi}), \bar{V}_{1}(\tilde{\Xi}), \bar{V}_{0}(\tilde{\Xi})$ according to Eq. (8).

The following parameters are chosen for the simulation of the Boolean models with different morphologies $\tilde{\Xi}_{\lambda, A, B, \phi}$ in the observation window $W=$ $[0,512]^{2}$. The typical grain $Z_{0}$ is a random rectangle:

rectangle sides: $A=e \cdot B, B \sim \Gamma\left(\frac{\mu^{2}}{\sigma^{2}}, \frac{\mu}{\sigma^{2}}\right)$,

where $\mu=\frac{\mathbb{E} V_{1}\left(Z_{0}\right)}{1+e}, \sigma^{2}=\frac{\mathbb{E} V_{2}\left(Z_{0}\right)}{e}-\mu^{2}$;

$\phi \sim U[0,2 \pi[$.

Fixed elongation ratio $e=1.5,2, \ldots, 9.5,10$.

Or random elongation ratio $\tilde{e} \sim \operatorname{Lognormal}(\mathbb{E} \tilde{e}, \mathbb{V} \tilde{e})$,

where $\mathbb{E} \tilde{e}=1.5,2, \ldots, 9.5,10 ; \mathbb{V} \tilde{e}=0.25$.

Test set $B_{r}(\tilde{x}), r=\frac{\mathbb{E} V_{1}\left(Z_{0}\right)}{4} ; \tilde{x} \sim U\left(\tilde{\Xi}_{\lambda, A, B, \phi} \cap W\right)$.

To get a stable numerical distribution of a local measure $N=50000$ realizations of a $\mathrm{BM}$ and $n_{l o c}=$ 500 positions of a test set are generated. The size of the test set was chosen based on the previous study of
Arns et al. (2005). Indeed, the smaller radius of the test set leads to a peak at 0 in the local perimeter measure $V_{1}(\tilde{\Xi}, \tilde{x})=\mathscr{H}^{1}\left(\partial \tilde{\Xi} \cap B_{r}(\tilde{x})\right)$ distribution, as the test set lie fully in the main set $\tilde{\Xi}$. On the other hand, the bigger size of the test set reveal more "global" morphology and the variance in the distributions of the local perimeter measure vanishes. An intermediate size of a test set should be chosen. For the studied datasets, in the hypothesis of a rectangular typical grain $Z_{0}$, and in view of the obtained values of the MFs Eq. (9)(10) of the typical grain, a test sizes $r=\frac{\mathbb{E} V_{1}\left(Z_{0}\right)}{4}$ is suggested, as it allows to distinguish better the pdfs of the local perimeter measure.

Figure 3 (1st row) shows the realizations of the Boolean models of rectangles with fixed elongation ratio $e=3$ and different area densities. Figure 4 (1st row) gives an example of realizations of the Boolean models of rectangles with the random elongation ratio which is log-normally distributed with $\mathbb{E} \tilde{\boldsymbol{e}}=3$ and different area densities. All BMs of rectangles used for the illustration of the results in this article are constructed with the parameters exposed in Eq. (11)-(16). The distributions of the local perimeter measure are compared for a system with the fixed and random elongation ratio in order to characterize the elongation ratio of the typical grain.

\section{MAXIMUM LIKELIHOOD ESTIMATION}

Let $\left(P_{1}, P_{2}, \ldots, P_{n}\right), n \geq 1$ be the realizations of the continuous real-valued random variable - the local perimeter measure $V_{1}(\tilde{\Xi}, \tilde{x})$. The subscript $n$ here reflects the total number of realizations of a random pair $(\tilde{\Xi}, \tilde{x})$. Explicitly,

$$
n=N \cdot n_{l o c},
$$

where $N$ stands for the number of realizations of an unknown Boolean model of rectangles $\tilde{\Xi}$, and $n_{l o c}-$ for the number of random locations $\tilde{x}$ of a test set. The likelihood of $e$ in view of $n$ observations $\left(P_{1}, P_{2}, \ldots, P_{n}\right)$ is defined as follows:

$$
L_{\tilde{\Xi}}(e)=\prod_{i=1}^{n} f_{e}\left(P_{i}\right) .
$$

where $f_{e}$ is the distribution of the variable $P$ parameterized by $e: f_{e}: \mathbb{R} \rightarrow \mathbb{R}_{+}$. As the locations of the test set are independent and identically distributed, so are the local perimeter measure values. Then, the maximum likelihood of the elongation ratio $e$ could be estimated as:

$$
\hat{e}=\underset{e}{\arg \max } L_{\tilde{\Xi}}(e) .
$$

For computational purposes, it is more convenient to use a log-likelihood function (a 
logarithmic transformation of the likelihood function is monotonously increasing and preserves the maximum). Thus, we obtain an optimization problem formulated as follows:

$$
\begin{aligned}
& \ln \left(L_{\tilde{\Xi}}(e)\right)=\sum_{i=1}^{n} \ln \left(f_{e}\left(P_{i}\right)\right), \\
& \hat{e}=\underset{e}{\arg \max } \sum_{i=1}^{n} \ln \left(f_{e}\left(P_{i}\right)\right) .
\end{aligned}
$$

In the present study only one parameter is to be optimized - the elongation ratio, it has been decided to use a direct search. For computational reasons, the elongation ratio $e$ was sampled with a step equal to 0.5 .

We quantify the error in the estimation of the elongation ratio for the different model morphologies by employing a relative mean error:

$$
R M E=\frac{1}{m} \sum_{i=1}^{m} \frac{\left|\hat{e}_{i}-e\right|}{e} .
$$

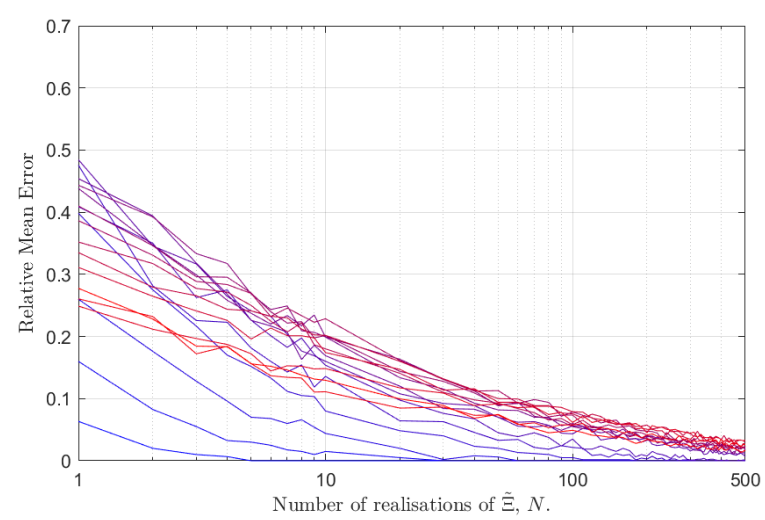

(a) For the BMs with the area density 0.4 in Fig. 3(b). Fixed elongation ratio $e=1.5,2, \ldots, 9.5,10$ (blue $\rightarrow$ red).

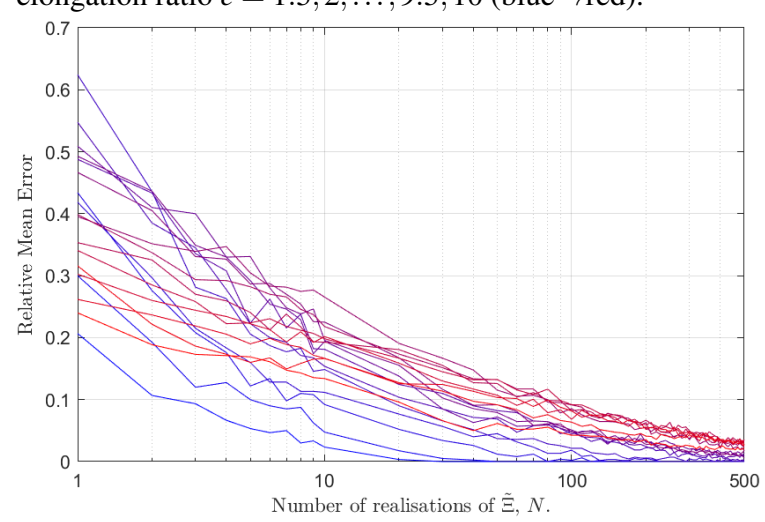

(b) For the BMs with the area density 0.9 in Fig. 4(c). Random elongation ratio expectation $\mathbb{E} \tilde{e}=1.5,2, \ldots, 9.5,10$ (blue $\rightarrow$ red).

Fig. 6: RME in the elongation ratio estimation for both systems of BMs of rectangles.
The same steps are followed for the estimation of the expectation of the elongation ratio $\mathbb{E} \tilde{e}$ in the case of the Boolean models of rectangles with the random elongation ratio.

\section{RESULTS}

As explained previously the global integral geometric measures could take the same values on very different structures. On the other hand, the local measures yield a supplementary information on the morphology of the spatial structure.

In Fig. 3 (2nd row) and 4 (2nd row) the correlation between the local perimeter measure and the typical grain elongation ratio is evidenced by comparing the probability density distributions of the local perimeter measure for the Boolean models of rectangles with all identical characteristics except for the elongation ratio.

Two datasets of Boolean models are used to validate the proposed method of the elongation ratio estimation. Tests are conducted on the simulated spatial structures - the realizations of the homogeneous Boolean models featuring equal global measures. For such a range of the BMs of rectangles with known elongation ratio (the elongation ratio distribution expectation in a case of the random elongation ratio), $N \geq 1$ realizations are generated and a local measure distribution is performed. Then, the elongation ratio is estimated using the method proposed in the previous section. To be statistically consistent, the procedure is repeated $m=100$ times for each $N$.

Figure 6 shows the relative mean error for the homogeneous Boolean models with parameters (11)(16). The estimation is more difficult for the Boolean models with grains of intermediate elongation ratio (see Fig. 7), and that despite the area density of the Boolean model. It is worth pointing out that even at high area density (0.9), when most grains overlap and the local analysis of the spatial structure is complicated, the prediction of the elongation ratio is still good. Indeed, the error in the estimation of $e$ for the BMs of rectangles is $<10 \%$ at $N=100$ realizations and $<5 \%$ at $N=500$ despite the density of the spatial structure. The relative mean error for two chosen configurations is explicitly given in Table 2 . 


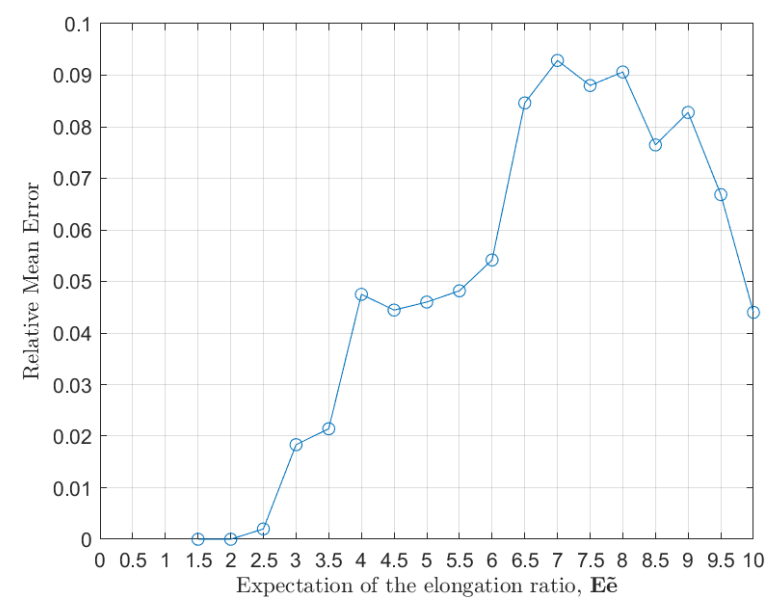

Fig. 7: Relative mean error of the elongation ratio estimation for the number of realizations $N=100$ at the highest area density 0.9 for a Boolean model of rectangles with the random elongation ratio. as long as the number of the $\mathrm{BM}$ realizations is big enough (error is $<10 \%$ at $N=100$ ).

In practice these typical grain elongation ratio analysis could for example be useful to control the pharmaceutical crystallization processes in order to assess the crystal growth rate (Ahmad et al., 2012). Next steps are the identification of the further relationships between the local measures distributions and the quantitative descriptors of the porous media (porosity, rugosity, tortuosity, etc.). A second prospect is to investigate other germ-grain models.

\section{REFERENCES}

Ahmad OS, Debayle J, Gherras N, Presles B, Fevotte G, Pinoli JC (2012). Quantification of overlapping polygonal-shaped particles based on a new segmentation method of in situ images during crystallization. J Electron Imaging 21.

CONCLUSION AND PERSPECTIVES Arns CH, Knackstedt MA, Mecke KR (2002). Characterising the morphology of disordered materials. Lect Notes Phys 600:37-74.

In this paper the local measures distribution estimation is applied to the particular RACS - a homogeneous Boolean model of rectangles uniformly randomly oriented and with a fixed or random elongation ratio. The correlation between the local perimeter measure and the typical grain elongation ratio is evidenced. Based on this correlation, a method has been developed to estimate the elongation ratio of the typical grain in a Boolean model. Under the hypothesis of a known shape of the typical grain (random rectangle in $\mathbb{R}_{+} \times \mathbb{R}_{+} \times[0,2 \pi[$ ), it has been demonstrated that it is possible to retrieve the elongation ratio of a rectangle despite the area density of the structure. Moreover, it has been shown that the nature of the elongation ratio (fixed or random) has a little influence on the accuracy of the estimation

Arns CH, Mecke J, Mecke K, Stoyan D (2005). Second-order analysis by variograms for curvature measures of two-phase structures. Eur Phys J B 47:397-409.

Baddeley AJ, Bárány I, Schneider R, Weil W (2006). Stochastic geometry: Lectures given at the C.I.M.E. Summer school held in martina franca, italy, september 13-18, 2004: Springer Berlin Heidelberg.

Baddeley AJ, Molchanov IS (1997). On the expected measure of a random set. In: Jeulin D, ed. Proceedings of the International Symposium on Advances in Theory and Applications of Random Sets (Fontainebleau, 1996). pp. 3-20.

\begin{tabular}{|c|c|c|c|c|c|c|c|c|c|c|c|c|c|c|c|c|c|c|}
\hline$e, \mathbb{E} \tilde{e}=$ & 1.5 & 2 & 2.5 & 3 & 3.5 & 4 & 4.5 & 5 & 5.5 & 6 & 6.5 & 7 & 7.5 & 8 & 8.5 & 9 & 9.5 & 10 \\
\hline $\bar{V}_{2}(\tilde{\Xi})=$ & \multicolumn{18}{|c|}{$\tilde{\Xi}:$ BM of rectangles with fixed elongation ratio } \\
\hline 0.2 & 0 & 0 & 0 & 0 & 0 & 0.3 & 0.3 & 0.3 & 0.5 & 0.7 & 0.5 & 1.5 & 2.3 & 2.1 & 2.2 & 1.8 & 1.5 & 1 \\
\hline 0.4 & 0 & 0 & 0 & 0 & 0 & 0.1 & 0.7 & 1.2 & 2.1 & 1.8 & 1.8 & 2.4 & 2.3 & 3 & 2.6 & 3.3 & 2.6 & 1.7 \\
\hline 0.9 & 0 & 0 & 0 & 0 & 0 & 0.3 & 0.6 & 0.9 & 2 & 1.7 & 2.8 & 2.6 & 3 & 2.9 & 3.8 & 3.9 & 2.2 & 2.6 \\
\hline $\bar{V}_{2}(\tilde{\Xi})=$ & \multicolumn{18}{|c|}{$\tilde{\Xi}: \mathrm{BM}$ of rectangles with random elongation ratio } \\
\hline 0.2 & 0 & 0 & 0 & 0 & 0 & 0.1 & 0 & 0.9 & 0.9 & 0.9 & 1.1 & 1.6 & 2.2 & 2 & 2.1 & 1.5 & 1.9 & 1 \\
\hline 0.4 & 0 & 0 & 0 & 0 & 0 & 0.4 & 0.3 & 1 & 1.2 & 1.9 & 2.4 & 2.4 & 1.8 & 2.9 & 3.1 & 2.8 & 3.1 & 1.3 \\
\hline 0.9 & 0 & 0 & 0 & 0 & 0 & 0.3 & 0.9 & 1.4 & 0.6 & 1.7 & 1.8 & 2.4 & 3.3 & 3.6 & 3.1 & 2.7 & 2.5 & 2.2 \\
\hline
\end{tabular}

Table 2: Relative mean errors (\%) of the elongation ratio estimation for BMs in Fig. 3 and Fig. 4 at N=500 realizations. 
Ballani F (2007). The surface pair correlation function for stationary boolean models. Adv Appl Probab 39:1-15.

Chiu SN, Stoyan D, Kendall WS, Mecke J (2013). Stochastic geometry and its applications, 3rd Ed. Chichester: Wiley.

Hadwiger H (1957). Vorlesungen über inhalt, oberfläche und isoperimetrie: Springer.

Hilfer R (2000). Local porosity theory and stochastic reconstruction for porous media. In: Mecke KR, Stoyan D, eds. Statistical Physics and Spatial Statistics: the art of analyzing and modeling spatial structures and pattern formation. Lect Notes Phys 554, pp. 203-41. Springer-Verlag, Berlin.

Klain DA, Rota G-C (1997). Introduction to geometric probability: Cambridge University Press.

Legland D, Kiêu K, Devaux M-F (2007). Computation of minkowski measures on $2 \mathrm{~d}$ and $3 \mathrm{~d}$ binary images. Image Anal Stereol 26:83-92.

Matheron G (1975). Random Sets and Integral Geometry. Wiley. New York.

Mecke KR (2000). Additivity, convexity, and beyond: applications of Minkowski Functionals in statistical physics. In: Mecke KR, Stoyan D, eds. Statistical Physics and Spatial Statistics: the art of analyzing and modeling spatial structures and pattern formation. Lect Notes Phys 554, pp. 111-84. Springer-Verlag, Berlin.

Miles RE (1976). Estimating aggregate and overall characteristics from thick sections by transmission microscopy. J Microsc-Oxford 107:227-33.

Molchanov I, Stoyan D (1994). Asymptotic properties of estimators for parameters of the boolean model. Adv Appl Probab 26:301-23.

Pinoli J-C (2014). Mathematical foundations of image processing and analysis, volume 2: John Wiley \& Sons.

Presles B, Debayle J, Pinoli JC (2012). Size and shape estimation of 3-d convex objects from their 2-d projections: Application to crystallization processes. J Microsc 248:140-55.

Rahmani S, Pinoli J-C, Debayle J (2017). Description of the symmetric convex random closed sets as zonotopes from their feret diameters. Mod. Stoch., Theory Appl. 3:325-64.

Santaló LA (1976). Integral Geometry and Geometric Probability, Addison-Wesley, Reading.

Schneider R (2014). Convex Bodies: The BrunnMinkowski Theory: Cambridge university press.

Schneider R, Weil W (2008). Stochastic and Integral Geometry, Probability and Its Applications, Springer Berlin Heidelberg.

Scholz C, Wirner F, Klatt MA, Hirneise D, SchröderTurk GE, Mecke K, Bechinger C (2015). Direct relations between morphology and transport in Boolean models. Phys Rev E Stat Nonlin Soft Matter Phys. 92:043023.

Sevink GJA (2007). Mathematical description of nanostructures with minkowski functionals. Nanostructured soft matter: Springer. pp 269-99.

Weil W (2001). Densities of mixed volumes for boolean models. Adv Appl Probab 33:39-60.

Zähle M (1982). Random-processes of hausdorff rectifiable closed-sets. Math Nachr 108:49-72. 